\title{
APPLICATION OF SENTINEL-1 MULTI-TEMPORAL DATA FOR CROP MONITORING AND MAPPING
}

\author{
R. Nasirzadehdizaji ${ }^{1, *}$, F. B. Sanli ${ }^{1}$, Z. Cakir ${ }^{2}$ \\ ${ }^{1}$ Dept. of Geomatic Engineering, Faculty of Civil Engineering, Yildiz Technical University, 34220 Esenler, Istanbul, Turkey - \\ rouhollah.nasirzadehdizaji@std.yildiz.edu.tr, fbalik@yildiz.edu.tr \\ ${ }^{2}$ Dept. of Geology, Faculty of Mines, Istanbul Technical University, 34469 Maslak, Istanbul, Turkey - ziyadin.cakir@itu.edu.tr
}

KEY WORDS: Sentinel-1, SAR, Backscatter, Time-series Analysis, Agricultural Monitoring, Crop Mapping

\begin{abstract}
:
The Synthetic Aperture Radar (SAR) technique superiority has provided various opportunities in agricultural studies mainly on crop monitoring and management. This has resulted in many different investigations and improvements in crop growth monitoring, crop disaster prediction and providing accurate information to precise farming. In this study, a time-series of Sentinel-1 SAR images were acquired throughout the agricultural season synchronously with the field measurements to investigate the temporal backscatter changes for different crop types. From the backscattering analysis, it was observed that each similar crop type in different test fields due to the distinct methods of irrigation and fertilization has shown different intensity values. However, they all follow a roughly identical backscattering trend during growing stages of the crop and useful information can be extracted, such as estimating irrigation and harvesting time according to the changes made in backscatters. In comparing with ascending orbit in VH and VV polarizations it has indicated that the homogeneity between SAR backscatters is high for each field with the same crop type in descending pass direction with VH polarization. In contrary, high-intensity values are recorded in VV polarization for entire crop types. It is also observed that polarimetric composite images for a different date are useful to roughly identify crop types, and validated with the application of classification methods in the study area. As preliminary results, it is demonstrated that SAR data provide useful information about crops status. Hence, Sentinel-1 SAR data are ideal preference due to its free availability and constant long-term data archive.
\end{abstract}

\section{INTRODUCTION}

Agriculture is in amongst important activities that have played a key role in providing food security to a growing population of the world (Soria-Ruiz, 2009). In this regard, sustainable food security is dependent on precise agricultural activities monitoring and the collection of accurate farming information (Canisus, 2017). Precision agriculture is a management strategy that integrates information and communication technologies with the agricultural industry. Therefore, the information of each component of the small area in a farm is used to adapt the type and amount of inputs in those areas in order to evaluate and manage the temporal and spatial variability more precisely. Satellite data are widely used to study and investigate agriculture activities changes as dynamic phenomenon over the time and in terms of quantitative and qualitative agricultural products, estimation of the planted area, the identification of certain types of crops, the growth stages and disaster prediction (Zhang, 1999).

Synthetic Aperture Radar (SAR) remote sensing system uses the microwave wavelength portion of the electromagnetic spectrum. Hence, the application of SAR data is very useful in agricultural monitoring due to the sensitivity of the microwave wavelength to the several characteristics of the crops. SAR is an active system independent of illumination sources to obtain data dayand-night and provide reliable and frequent imaging, independently of cloud coverage, as a key factor in the context of agricultural applications (Moreira, 2013). Microwaves can penetrate through clouds where the cloudy sky is a serious obstacle to the application of optical images particularly in a rainy climate. The SAR system has sensitivity to the physical morphology and the geometrical characteristics of the land surface and cover (soil roughness, moisture, vegetation structure, etc.). Therefore, radar sensors collect the echoes of the backscattered signal in a sequential way thus very different to that of optical satellite data, which measure reflected solar light in visible and infrared wavelengths (Wooding, 1995).

The two-polar-orbiting satellite constellation (Sentinel-1A and 1B) equipped with a dual-polarized SAR C-band at a $20 \mathrm{~m}$ spatial resolution offers a 6 days repeat frequency in a different operational mode which enables users to access freely available of a constant long-term data archive for applications requiring long-range time-series (Panetti, 2012).

This study investigates the potential of Sentinel-1 polarimetric SAR backscatter data in an agricultural area for growth monitoring of different crop types (maize, sunflower, wheat and potato) and crop mapping using that polarimetric composite of images which are produced from multi-temporal analysis.

\section{MATERIALS AND METHODOLOGY}

\subsection{Study site description and field surveys}

The Konya basin in the central Anatolia Turkey, $\left(38^{\circ} 40^{\prime} \mathrm{N}, 32^{0}\right.$ $26^{\prime}$ E) which is about $40 \mathrm{~km}^{2}$, was selected for field measurements and satellite images collection (Figure 1). The study area has partially flat topography with a gentle slope (2\%-

\footnotetext{
* Corresponding author
} 
$6 \%$ ) and, the fields' sizes are ranging between 0.5 and the 18 ha. The soil type is mainly Reddish Brown and Brownish soils in the study region according to the Ministry of Agriculture and Forestry of Turkey. The soil texture consists of well to moderately well drained sand and sandy loam, and imperfectly to poorly drained clay and silt clay loam.

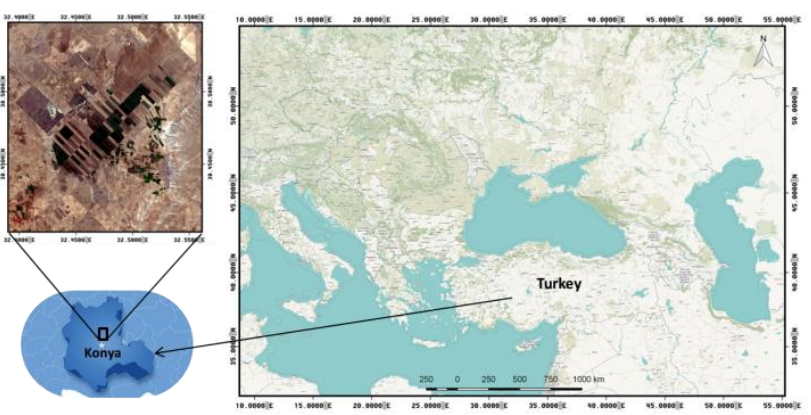

Figure 1. The location map of the study area

The study site has an arid to semi-arid predominant weather condition and land use type is mainly agricultural land. Due to the extent and dense of agricultural activities, the demand for water consumption for irrigation is increasing in the region. Insitu measurements for under investigating site was conducted for maize, sunflower wheat and potato fields in the springsummer agricultural season of the year 2016. Among the various agricultural products in the study area due to their different structures maize, sunflower, wheat and potato are four investigated crops patterns. Maize, sunflower and potato based on filed measurements, generally are cultivated at the beginning of April to mid-May and harvested in August or the beginning of September in this study area. Winter wheat is seeded in previous October and harvested in July. During the field work, crop variables and parameters that indicate the growth rate of the crops including crop height and canopy coverage are recorded. In addition, field characteristics such as soil properties and irrigation status were collected in the field surveys. The in-situ measurements were conducted according the four main development periods including leaf development, stem elongation, heading and flowering stages of the crops that has been defined under Biologische Bundesanstalt, bundessortenamt und CHemische industrie Sacle $(\mathrm{BBCH}-$ Sacle) (Meier, 2001). Figure 2 indicates the crop calendar for maize, sunflower, wheat and potato in the study area.

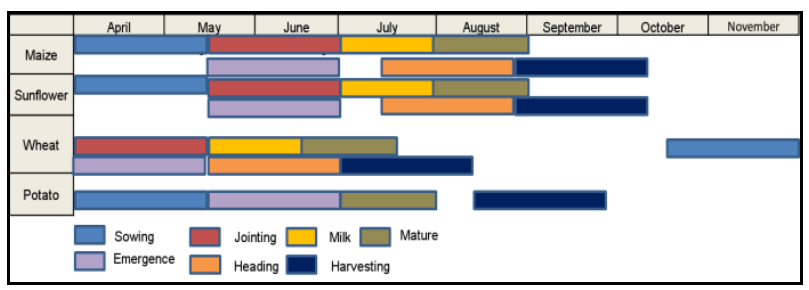

Figure 2. Seasonal maize, sunflower, wheat and potato calendar in the study area

\subsection{Sentinel-1 SAR data statement and processing}

Sentinel-1 Interferometric Wide swath (IW) mode images were acquired in both ascending and descending orbit pass directions. We used Level-1 C-band Ground Range Detected (GRD) products that consist of focused SAR data which has been detected, multi-looked and projected to ground range using an Earth ellipsoid model such as WGS84. The satellite obtains data with dual-polarization (VV and $\mathrm{VH}$ ) backscatter. For the intensity analysis of SAR backscatter to crops, a time series of 24 remotely sensed Sentinel-1 SAR data in ascending and 23 data in descending pass direction were acquired starting from 02 May to 24 September in 2016. Processing steps as radiometric calibration, speckle filtering, topographic correction using SRTM second data and co-registration were performed with open source tools of Sentinel Application Platform (SNAP) software (SNAP Development Team, 2019). As a last step, pixel digital numbers were converted to sigma nought in decibel $(\mathrm{dB})$. Spatial subset operation is applied to resize the images to reduce the amount of processing time. Flowchart of multi-temporal Sentinel-1 SAR data processing is shown in Figure 3.

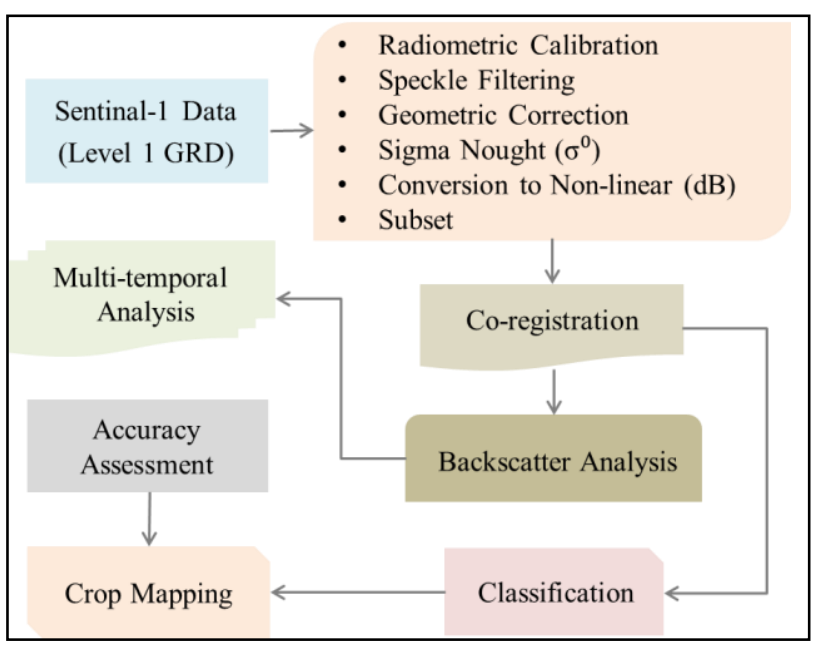

Figure 3. Workflow of multi-temporal SAR analysis

As a post-processing step, Maximum Likelihood Classification (MLC) method is used for crop classification of SAR dataset to map the multi-temporal coverage of the seasonal crops in the study area. Six different classes consist of Potato, Sunflower, Maize, Wheat, Uncultivated and Bare Soil used to provide reliable crop map. Overall accuracy and kappa coefficient are calculated from confusion matrix to evaluate the quality of the crop map. Classification process was performed using SemiAutomatic Classification Plugin (SCP) as free open source plugin for QGIS (Congedo, 2016; QGIS Development Team, 2019).

\section{RESULTS AND DISCUSSIONS}

\subsection{SAR backscattering analysis}

The backscatters of individual pixels were determined and the correlation between the field measurements was evaluated by analysing multi-temporal SAR images for each field with different crop types. Then the backscatter signature of the selected individual pixels for all patterns in the same plot as well as backscattering value changes for each crop types with different fields during the timeframe of the study were interpreted. Figure 4 shows the variation of the backscatter value of each pattern in the timeframe of the study in ascending and descending orbit pass modes in $\mathrm{VH}$ and $\mathrm{VV}$ polarizations. While the backscatter values of selected pixels for each crop types change between $-11 \mathrm{~dB}$ and $-26 \mathrm{~dB}$ in $\mathrm{VH}$ polarization in ascending orbit direction, the values vary between $-4 \mathrm{~dB}$ and - 
$19 \mathrm{~dB}$ in VV polarized images in the same orbit pass. These values show variation between $-11 \mathrm{~dB}$ and $-27 \mathrm{~dB}$ in $\mathrm{VH}$ and $4 \mathrm{~dB}$ and $-18 \mathrm{~dB}$ in $\mathrm{VV}$ polarizations in descending pass mode. The backscatter results show that the satellite orbit pass directions did not affect the intensity values; whereas, in different polarizations change in values is considerable.

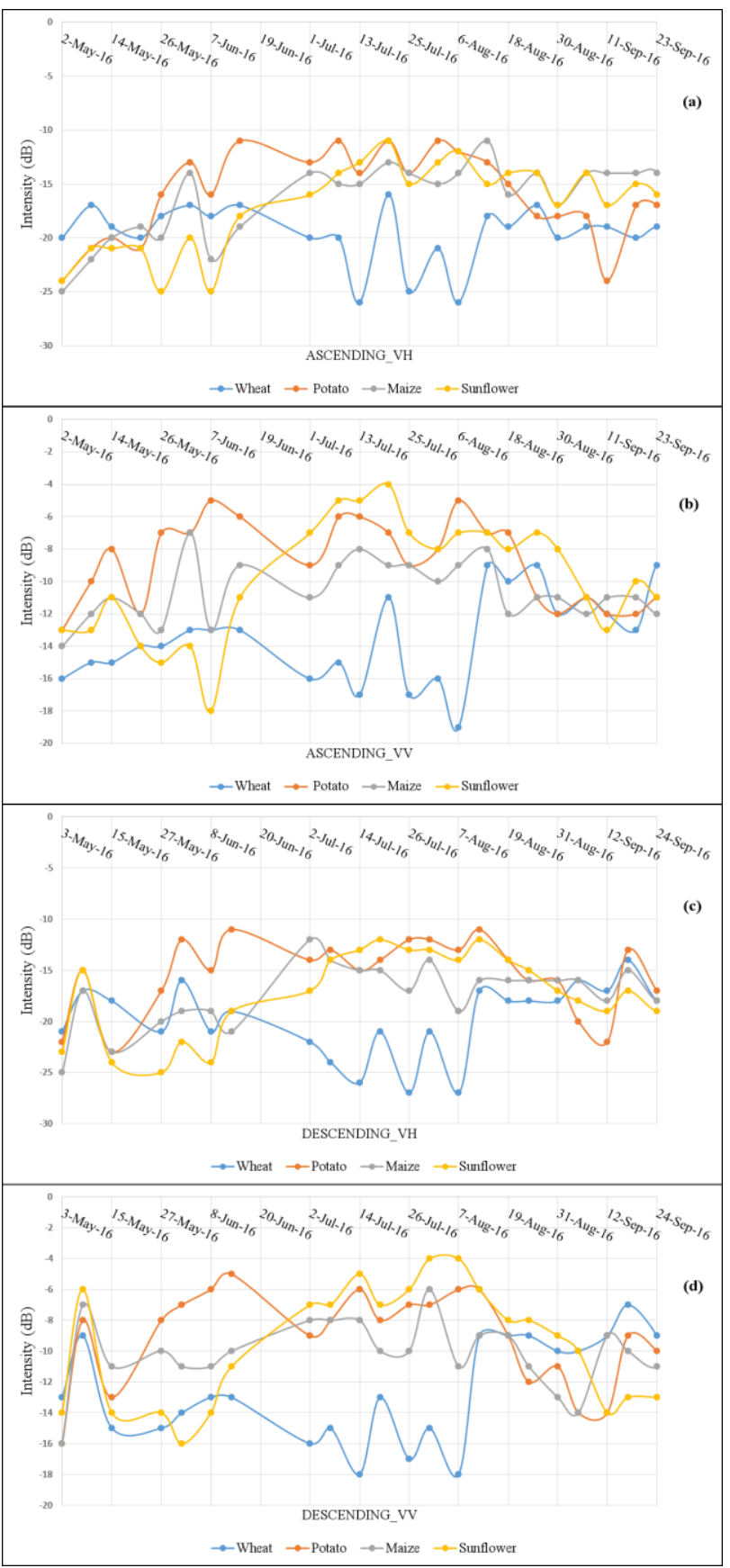

Figure 4. The backscatter value of maize, sunflower, wheat and potato on multi-temporal Sentinel-1 images, $(a, b)$ ascending and $(\mathrm{b}, \mathrm{c})$ descending in $\mathrm{VH}$ and $\mathrm{VV}$ polarization respectively

In Figure 4, backscatter value of all crop types at the early stages of the crops present a relatively homogenous intensity values. Whereas in the growing stages (mid stage) of the crops, due to differences in physical structure of the crops and the sensitivity of the SAR to the geometrical characteristics of the patterns, each crop types has high differences in backscatter values. It can be interpreted that in later stages of the crops there is a similarity in the backscatter value. According the field measurements, this point shows the end of the heading and beginning of the harvesting time. Time-series backscatter analysis gives very useful information when one crop type in various fields is intended to be observed in terms of the monitoring programs and management practices. The results also indicate that there is relationship between Sentinel-1 SAR backscatter values and crop variables such as crop height and crop coverage during the different phonological stages (Nasirzadehdizaji, 2019).

\subsection{Crop mapping}

In addition to the multi-temporal SAR backscattering analysis, polarimetric composite of the images of the different polarization over time without classification methods may also provide beneficial information regarding the identification of crop types. Figure 5 displays the polarimetric color composite images of Sentinel-1 in three observation times and each pin in the image represents one crop type. Identification of crop types is validated with the application of classification methods in the study area.

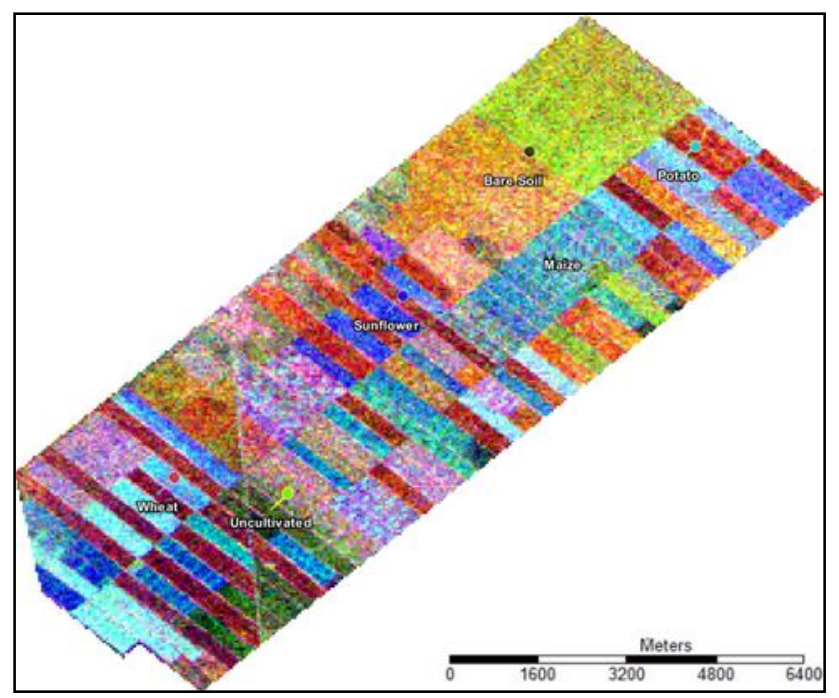

Figure 5. RGB color composite using multi-temporal Sentinel-1 SAR data in descending pass direction (R: 2016.05.15_VH_dB, G: 2016.06.01_VV_dB, B: 2016.07.31_VH_dB)

\subsection{Accuracy assessment of crop classification}

The classification has been done based on SAR backscatter values and their temporal changes in each class. Confusion (error) matrix (Table 1) was applied to evaluate the accuracy of the multi-temporal SAR-derived crop map and determination of the accuracy of the classification process. The confusion matrix just compares the reference points (test data) to the classified points (training data). Overall, produce and user accuracies, Kappa coefficient, the commission error and the omission error for each class were calculated from confusion matrix (Table 2). Crop map were produced after pixel-based image classification for multi-temporal SAR color composite image (Figure 6). The results showed that high overall accuracy of $88 \%$ and Kappa coefficient of 0.83 has been obtained (Figure 7). 


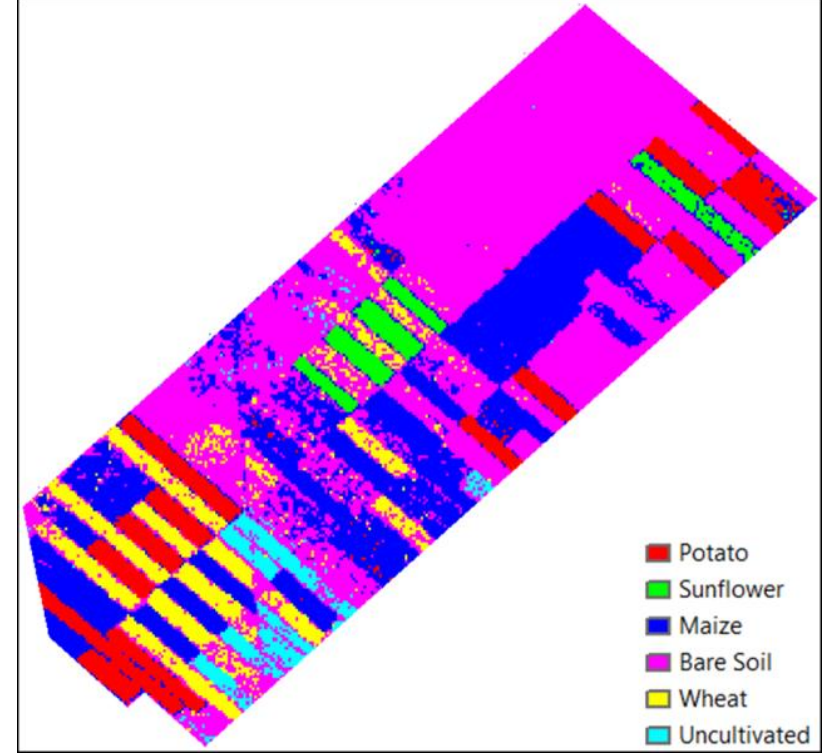

Figure 6. Crop classification of SAR dataset (MLC)

\begin{tabular}{|c|c|c|c|c|c|c|c|}
\hline Class & Potato & Sunflower & Maize & Wheat & Uncultivated & Bare Soil & Total \\
\hline Potato & 279 & 1 & 36 & 0 & 0 & 1 & 317 \\
\hline Sunflower & 27 & 105 & 56 & 1 & 0 & 0 & 189 \\
\hline Maize & 39 & 18 & 375 & 0 & 1 & 7 & 440 \\
\hline Wheat & 0 & 1 & 1 & 170 & 9 & 72 & 253 \\
\hline Uncultivated & 0 & 0 & 5 & 10 & 141 & 33 & 189 \\
\hline Bare Soil & 0 & 0 & 3 & 2 & 4 & 1384 & 1393 \\
\hline Total & 345 & 125 & 476 & 183 & 155 & 1497 & 2781 \\
\hline
\end{tabular}

Table 1. Maximum Likelihood classification confusion matrix

\begin{tabular}{|c|c|c|c|c|}
\hline Class & Commission & Omission & Producer Accuracy & User Accuracy \\
\hline Potato & 11.99 & 19.13 & 80.87 & 88.01 \\
\hline Sunflower & 44.44 & 16 & 84 & 55.56 \\
\hline Maize & 14.77 & 21.22 & 78.78 & 85.23 \\
\hline Wheat & 32.81 & 7.1 & 92.9 & 67.19 \\
\hline Uncultivated & 25.4 & 9.03 & 90.97 & 74.6 \\
\hline Bare Soil & 0.65 & 7.55 & 92.45 & 99.35 \\
\hline
\end{tabular}

Table 2. Percentages of errors of commission, errors of omission, Producer's and User's accuracies for each class

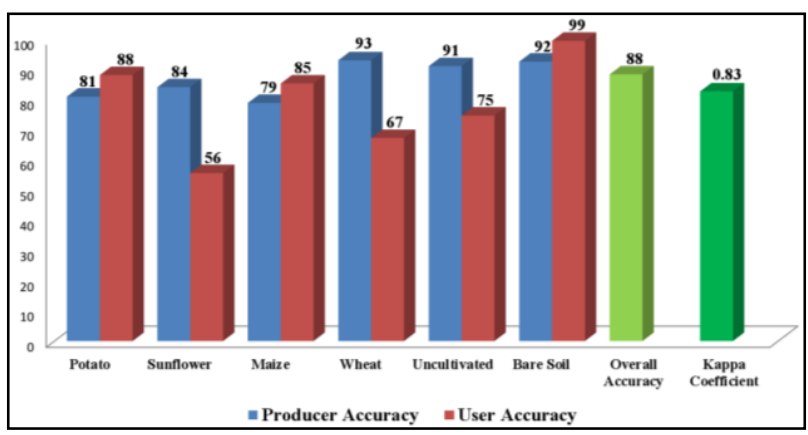

Figure 7. Producer's, User's and Overall accuracies and Kappa coefficient assessment of multi-temporal Sentinel-1 SAR data (percentages are rounded to the nearest decimal)

\section{CONCLUSION}

Backscatter values for each crop types (i.e., maize, sunflower, wheat and potato) over the time were determined and the relation between the intensity values and the data obtained from filed campaign was evaluated. Due to the different methods of irrigation and fertilization and the differences in crops physical geometry during the growing stages, different backscatter values are observed for different types of crops. However, at the later stages of the crops growth, due to reaching the harvesting time, similar backscatter values are observed. Moreover, the backscatter signature in timeframe varies considerably over different areas and thus it may not be possible to drive a unique backscatter signature. That is valid for the same crop in all areas although this distribution gives an indication of the differences in backscatter for different crop over time.

In this study, Sentinel-1 SAR backscatters over the time and throughout the agricultural season in the study area was evaluated. The relationship between ascending and descending pass directions with $\mathrm{VH}$ and VV polarization was investigated. It was demonstrated that early and later stages of crops growth have relatively similar correlation in backscatter values and opposite to this, mid stages showed lower similarity due to geometrical characteristics of the different crop types. In addition, it has indicated that the homogeneity between SAR backscatters is high for each field with the same crop type in descending pass direction with VH polarization. In conclusion, using the multi-temporal Sentinel-1 SAR data for the agriculture monitoring system which may play an important role for the accurate crop assessment is an ideal preference due to its free availability and a constant long-term data archive.

\section{REFERENCES}

Canisus, F., Shang, J., Liu, J., Huang, X., Jiao, X., Geng, X., Kovacs, J.M., Walters, D., 2017. Tracking crop phenological development using multi-temporal polarimetric Radarsat-2 data. Remote Sens. Environ.

Congedo L., 2016. Semi-Automatic Classification Plugin Documentation.http://dx.doi.org/10.13140/RG.2.2.29474.02242 $/ 1$.

Meier, U., 2001. Growth Stages of Mono- and Dicotyledonous Plants; BBCH Monograph, Federal Biological Research Centre for Agriculture and Forestry: Bonn, Germany, p. 158.

Moreira, A., Prats, P., Younis, M., Krieger, G., Hajnsek, I., Papathanassiou, K., 2013. A Tutorial on Synthetic Aperture Radar. IEEE Geoscience and Remote Sensing Magazine, march 2013.

Nasirzadehdizaji, R., Sanli F. B., Abdikan, S., Cakir, Z., Sekertekin, A., Ustuner, M., 2019. Sensitivity Analysis of Multi-Temporal Sentinel-1 SAR Parameters to Crop Height and Canopy Coverage. Appl. Sci. 9(4), 655. https://doi.org/10.3390/app9040655.

Panetti, A., Torres, R., Lokas, S., Bruno,C., Croci, R., L'Abbate, M., Marcozzi, M., Pietropaolo, A., Venditti, P., 2012. GMES Sentinel-1: Mission And Satellite System Overview. EUSAR 2012. 162-165. 
QGIS Development Team, 2019. QGIS Geographic Information System, Open Source Geospatial Foundation Project. http://qgis.osgeo.org (16 June 2019).

SNAP Development Team, 2019. Sentinel Application Platform Software (SNAP). http://step.esa.int/ main/toolboxes/snap/ (23 April 2019).

Soria-Ruiz, J., Fernandez-Ordonez, Y., McNairn, H., 2009. Corn Monitoring and Crop Yield Using Optical and Microwave Remote Sensing. Geosci. Remote Sens., 405-419.

Wooding, M.G., Attema, E., Aschbacher, J., Borgeaud, M., Cordey, R.A., de Groof, H., Harms, J., Lichtenegger, J., Nieuwenhuis, G., Schmullius, C., and Zmuda, A.D. 1995. Satellite radar in agriculture: experience with ERS-1. ESA Special Publication SP-1185. 72 pp.

Zhang, W., Yan, T., 1999. Analysis of advantage on radar remote sensing for agricultural application. In Proceedings of the Asian Conference on Remote Sensing, Hong Kong, China, 22-25 November 1999. 